\title{
High-frequency capability of Schottky-barrier carbon nanotube FETs
}

\author{
L.C. Castro ${ }^{a}$, D.L. Pulfrey ${ }^{b}$ and D.L. John ${ }^{c}$ \\ Department of Electrical Engineering, University of British Columbia, \\ 2356 Main Mall, Vancouver, BC, V6T 1Z4, Canada \\ a leonardo@ece.ubc.ca, ${ }^{b}$ pulfrey@ece.ubc.ca, ${ }^{c}$ davej@ece.ubc.ca
}

Keywords: carbon nanotube field-effect transistor, small-signal properties, high-frequency figures of merit, resonance.

\begin{abstract}
The high-frequency capability of carbon nanotube field-effect transistors is investigated by simulating the small-signal performance of a device with negative-barrier Schottky contacts for the source and drain, and with a small, ungated region of nanotube between the end contacts and the edge of the wrap-around gate electrode. The overall structure is shown to exhibit resonant behaviour, which leads to a significant bias dependence of the small-signal capacitances and transconductance. This could lead to high-frequency figures of merit $\left(\mathrm{f}_{\mathrm{T}}\right.$ and $\left.\mathrm{f}_{\max }\right)$ in the terahertz regime.
\end{abstract}

\section{Introduction}

Carbon nanotube field-effect transistors (CNFETs) are predicted to have superior DC characteristics to those of foreseeable silicon MOSFETs [1,2]. Experimentally, impressive values for drain current and transconductance have already been reported in prototype devices [3]. However, the AC performance of CNFETs has not yet received much attention [4], and the likelihood of high-frequency operation needs to be established. Here, we take a step in this direction by predicting two useful figures of merit for high-frequency transistors, namely: the unity current-gain and unity power-gain frequencies, $f_{\mathrm{T}}$ and $\mathrm{f}_{\max }$, respectively.

We employ the standard small-signal method [5], abetted by a self-consistent SchrödingerPoisson solver [6], in order to obtain preliminary estimates of these valuable figures of merit. In particular, we consider the cylindrically gated device depicted in Fig. 1. The CNFET geometry consists of a nanotube with radius $r_{t}$, coated by an insulator of thickness $t_{\text {ins }}$, wrapped by a cylindrical gate of thickness $t_{\mathrm{g}}$, and capped at the ends by planar source and drain contacts of radius $t_{i n s}+t_{g}+r_{t}$. These end contacts are separated longitudinally from the gate by a distance $L_{g a p}$.

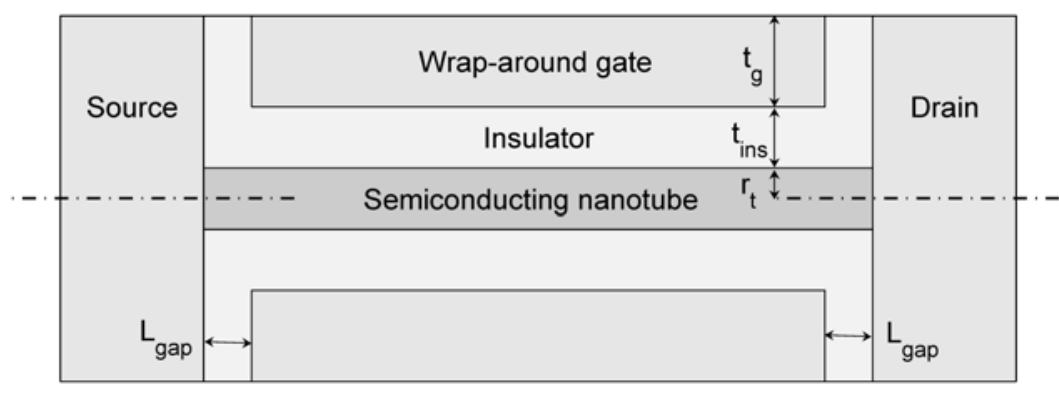

Figure 1. Structure of the modeled CNFET.

\section{Model}

Since the CNFET is much like a traditional field-effect transistor, we may employ the standard equivalent circuit model for this device [5], where the small-signal parameters themselves, such 
as the transconductance and the various transcapacitances, are computed by taking numerical derivatives based on the results of self-consistent DC charge-voltage calculations [6,7]. Through our use of a Schrödinger-Poisson solver for these DC results, we are able to include the effects of geometry, quantum capacitance, and spatial non-uniformity of the charge in our calculations.

While we have previously presented results for the intrinsic $f_{T}$ with only three circuit elements [7], here we consider the additional effects of series resistances associated with each of the terminals, and we include the remaining capacitance components. This allows us to compute the extrinsic $\mathrm{f}_{\mathrm{T}}$, and also to consider $\mathrm{f}_{\max }$. For clarity, we include the standard equivalent circuit in Fig. 2, where we note that the subscripts $\mathrm{s}$, $\mathrm{d}$ and $\mathrm{g}$ refer to the source, drain and gate, respectively. In the usual way, we consider the $f_{T}$ and $f_{\max }$ expressions, which may be arrived at by extrapolating the characteristic decay in gain, from its value at an appropriate low frequency, to the $0 \mathrm{~dB}$ point.

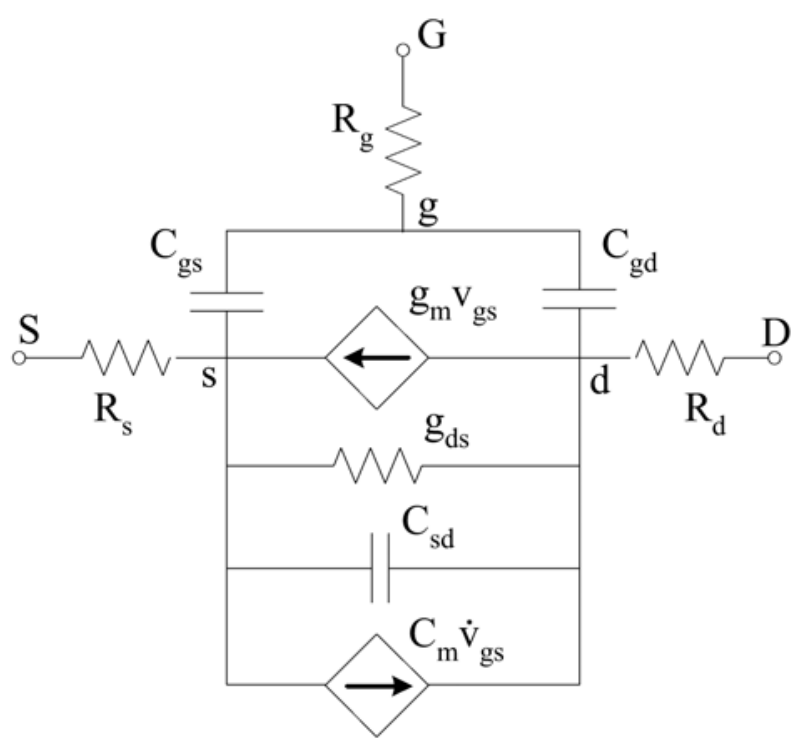

Figure 2. Small signal equivalent circuit. The transcapacitance $C_{m}=C_{d g}-C_{g d}$.

\section{Results and Discussion}

The model device in this study employs a $(16,0)$ nanotube of radius $0.63 \mathrm{~nm}$, length $30 \mathrm{~nm}$, bandgap $0.62 \mathrm{eV}$, unity relative permittivity, and effective mass $0.06 \mathrm{~m}_{0}$, where $\mathrm{m}_{0}$ is the free electron rest mass. Moreover, $t_{\text {ins }}$ is $2.5 \mathrm{~nm}, t_{\mathrm{g}}$ is $3 \mathrm{~nm}$, the insulator permittivity is 25 as is appropriate for zirconia [8], and $\mathrm{L}_{\text {gap }}$ is $5 \mathrm{~nm}$ for this initial investigation. The work function of the nanotube is taken to be $4.5 \mathrm{eV}$, while that of the end-contacts is $3.9 \mathrm{eV}$, yielding negativeSchottky barrier, n-type transistor operation. All results are for a drain-source voltage of $0.5 \mathrm{~V}$.

Fig. 3 illustrates the intrinsic parameters for our device. The oscillatory peaks in the capacitances and transconductance are related to the formation of quasi-bound states in the short channel [7,9]. With the modulation of the applied voltage, the states, indicated by the bright patches in Fig. 4, are shifted in energy by band bending in the channel. As they cross the source or drain Fermi level, they become populated, and we obtain peaks in the charge accumulation and, consequently, in the capacitances. Since the charge accumulation affects the amount of band bending in the channel through our self-consistent DC calculations, we also see peaks in the transconductance $\mathrm{g}_{\mathrm{m}}$. The $\mathrm{g}_{\mathrm{m}}$ behaviour is complicated due to its dependence both on the charge and on the Fermi distributions at the injecting contacts.

In Fig. 5, we present our main results, the predictions of $f_{T}$ and $f_{\max }$ for our model CNFET. Fig. 5(a) shows $f_{T}$ for $R_{s}=R_{d}$ set to $1 \mathrm{k} \Omega, 10 \mathrm{k} \Omega$, and $100 \mathrm{k} \Omega$, and we recall that $R_{g}$ has no influence on this figure of merit. Note that the values of the parasitic resistors $R_{s}$ and $R_{d}$ are 
chosen to be comparable to the contact resistance that results from mode constriction when carriers pass from a many-moded material to a material with only a few modes [10]. In the carbon nanotube case, we consider the lowest two degenerate modes in energy for an equivalent contact resistance of around $6-7 \mathrm{k} \Omega$. Note that the contact resistance is automatically included in our self-consistent DC calculations, so an explicit resistor is not needed to represent it in the equivalent circuit.. Thus, the resistors shown in Fig. 2 are solely parasitic. It is evident that the maximum value of $f_{T}$ occurs at the first peak in $g_{m}$. Turning now to $f_{\max }$, shown in Fig. 5(b), we focus on the effect of $R_{g}$, and show results for $R_{g}$ set to $100 \Omega, 1 \mathrm{k} \Omega$, and $10 \mathrm{k} \Omega$, with $R_{s}=R_{d}=$ $10 \mathrm{k} \Omega$. Again, a pronounced peak coincides with the first peak in $\mathrm{g}_{\mathrm{m}}$.
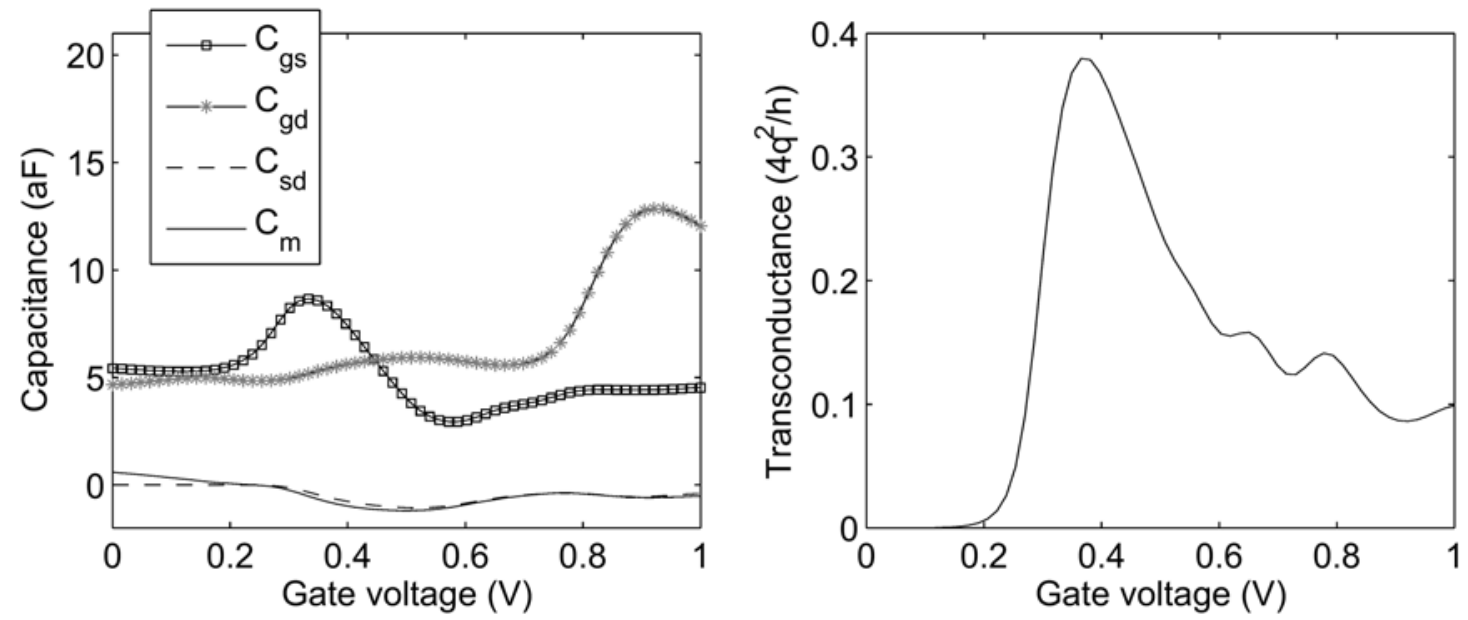

Figure 3. Capacitances and transconductance for the model device.

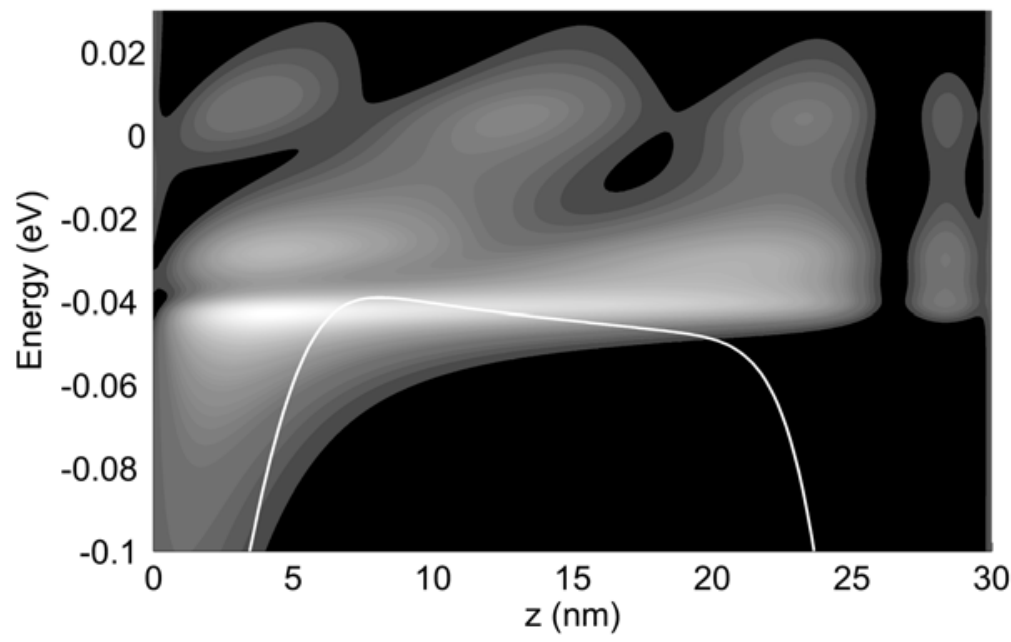

Figure 4. Charge density for the model device subject to a gate-source voltage of $0.38 \mathrm{~V}$.

Brighter patches indicate higher charge density, while a portion of the conduction band edge is shown by the white line. The energy values are referenced to the source Fermi level. 

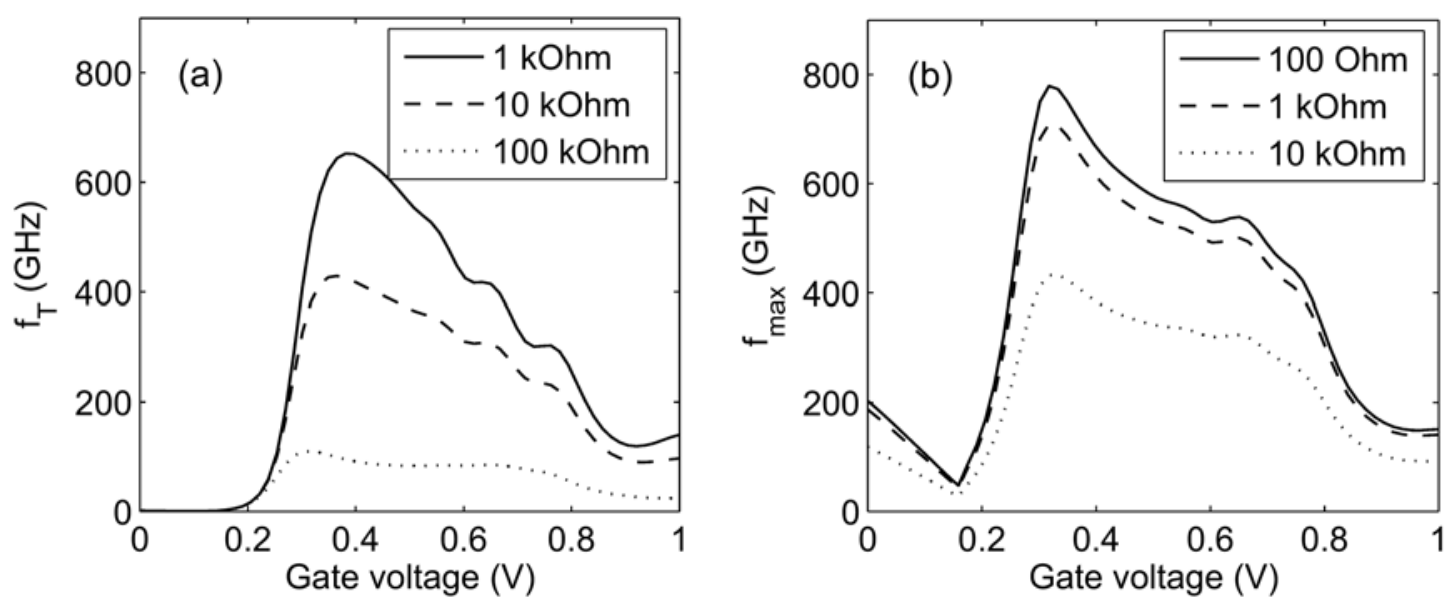

Figure 5. Extrapolated figures of merit: (a) $f_{T}$ with $R_{S}=R_{d}$ set to $1 \mathrm{k} \Omega$ (solid), $10 \mathrm{k} \Omega$ (dashed), and $100 \mathrm{k} \Omega$ (dotted), and (b) $\mathrm{f}_{\max }$ with $\mathrm{R}_{\mathrm{s}}=\mathrm{R}_{\mathrm{d}}=10 \mathrm{k} \Omega$ for $\mathrm{R}_{\mathrm{g}}$ set to $100 \Omega$ (solid), $1 \mathrm{k} \Omega$ (dashed), and $10 \mathrm{k} \Omega$ (dotted).

\section{Conclusions}

From this simulation of the high-frequency performance of CNFETs, it can be concluded that, in short-channel devices, with negative-barrier Schottky contacts for the source and drain, and with very short ungated regions, and where coherent transport is possible, resonance effects can lead to a strong bias dependence of the high-frequency figures of merit, $\mathrm{f}_{\mathrm{T}}$ and $\mathrm{f}_{\max }$. At the resonance peaks, these frequencies may reach the $\mathrm{THz}$ level.

\section{References}

[1] L.C. Castro, D.L. John and D.L. Pulfrey: Smart Mater. Struct. Accepted Aug. 13, 2004. [Online.] Available: http://nano.ece.ubc.ca/pub/publications.htm.

[2] J. Guo, M. Lundstrom and S. Datta: Appl. Phys. Lett. Vol. 80 (2002), p. 3192.

[3] A. Javey, J. Guo, Q. Wang, M. Lundstrom and H. Dai: Nature Vol. 424 (2003), p. 654.

[4] D.V. Singh, K.A. Jenkins, J. Appenzeller, D. Neumayer, A. Gill and H.-S.P. Wong: IEEE Trans. Nanotechnol. Vol. 3 (2004), p. 383.

[5] Y.P. Tsividis: Operation and Modeling of the MOS Transistor, Chapter 9, (McGraw-Hill, Toronto 1987).

[6] D.L. John, L.C. Castro, P.J.S. Pereira and D.L. Pulfrey: Proc. NSTI Nanotech Vol. 3 (2004), p. 65.

[7] L.C. Castro, D.L. John, D.L. Pulfrey, M. Pourfath, A. Gehring and H. Kosina: IEEE Trans. Nanotechnol. Submitted Nov. 16, 2004.

[8] A. Javey, R. Tu, D. Farmer, J. Guo, R. Gordon and H. Dai: Nano Lett. Vol. 5 (2005), p. 345 .

[9] D.L. John, L.C. Castro and D.L. Pulfrey: J. Appl. Phys. Vol. 96 (2004), p. 5180.

[10] S. Datta: Electronic Transport in Mesoscopic Systems (Cambridge University Press, New York 1995). 